\title{
Risk of ischemic stroke in patients with prostate cancer receiving androgen deprivation therapy in Taiwan
}

\author{
Kuang-Ming Liao ${ }^{1 \dagger}$, Yaw-Bin Huang ${ }^{2,3}$, Chung-Yu Chen ${ }^{2,34^{*}}$ (D) and Chen-Chun Kuo ${ }^{3+}$
}

\begin{abstract}
Background: Androgen deprivation therapy (ADT) in the treatment of prostate cancer may be associated with an increased risk of thromboembolic disease. The aim of our study was to investigate the association of ADT in the treatment of prostate cancer with ischemic stroke risk.

Methods: We identified individuals older than 20 years of age who were newly diagnosed with prostate cancer between January 1, 2005, and December 31, 2012. Patients who experienced ischemic stroke or transient ischemic stroke before the index date were excluded. Patients who received at least one prescription for ADT within 6 months were defined as the ADT user group. Patients who did not receive at least one prescription for ADT within 6 months were defined as the ADT nonuser group. The patients were followed until the first occurrence of one of the primary outcome measures (ischemic stroke or death) or until December 31, 2013. The primary composite outcome was the time to any cause of death or ischemic stroke.

Results: There was no significant difference in the primary composite outcomes in the prostate cancer patients between the ADT user and nonuser groups. Prostate cancer patients who received ADT had a higher mortality rate than those who were not treated with ADT, and the adjusted hazard ratio was 1.907 (95\% confidence interval: 1.278-2.844; $P=0.0016$ ) after adjusting for age, comorbidities and comedication use.

Conclusion: ADT in the treatment of prostate cancer may not be associated with an increased risk of ischemic stroke. The differences in thromboembolic effects in cardiovascular disease and ischemic stroke secondary to ADT should be further discussed and evaluated prospectively.
\end{abstract}

Keywords: Androgen deprivation therapy, Ischemic stroke, Prostate cancer

\section{Background}

Androgen deprivation therapy (ADT) is the key treatment for advanced prostate cancer patients or patients with high serum prostate-specific antigen levels with intermediate or high-risk prostate cancer even without imaging findings or other evidence of disseminated disease $[1,2]$.

\footnotetext{
* Correspondence: jk2975525@hotmail.com

${ }^{\dagger}$ Kuang-Ming Liao and Chen-Chun Kuo indicates equal contribution in this study to the first author

${ }^{2}$ School of Pharmacy, Master Program in Clinical Pharmacy, Kaohsiung Medical University, No. 100, Shihcyuan 1st Rd., Sanmin District, Kaohsiung City 80708, Taiwan, Republic of China

${ }^{3}$ Department of Pharmacy, Kaohsiung Medical University Hospital, Kaohsiung, Taiwan

Full list of author information is available at the end of the article
}

Despite the frequently dramatic potential benefits after treatment, ADT has side effects that can greatly impact a patient's quality of life and may necessitate changing the treatment strategy. These side effects include sexual dysfunction [3], increased risk of bone fractures due to decreased bone mineral density [4], hot flashes [5], reduced muscle strength, decreased lean body mass, increased fat mass, decreased insulin sensitivity [6] and potential harm to the cardiovascular system [7].

There are existing conflicting reports regarding the effect of ADT on cardiovascular disease. Saigal et al. showed that ADT is associated with cardiovascular mortality and may decrease survival in patients with low-risk disease [8].

(c) The Author(s). 2019 Open Access This article is distributed under the terms of the Creative Commons Attribution 4.0 International License (http://creativecommons.org/licenses/by/4.0/), which permits unrestricted use, distribution, and reproduction in any medium, provided you give appropriate credit to the original author(s) and the source, provide a link to the Creative Commons license, and indicate if changes were made. The Creative Commons Public Domain Dedication waiver (http://creativecommons.org/publicdomain/zero/1.0/) applies to the data made available in this article, unless otherwise stated. 
Tsai et al. reported that ADT significantly increased mortality due to cardiovascular disease in patients who underwent radical prostatectomy for localized prostate cancer [9].

Nanda et al. found that ADT increased the risk of allcause mortality in patients with a history of congestive heart failure or myocardial infarction, but this effect was not observed in patients without comorbidities or those with a single coronary artery risk factor [10].

However, a systematic review and meta-analysis found that ADT did not increase the risk of cardiovascular death and that ADT reduced prostate cancer-specific mortality and all-cause mortality [11]. A recent metaanalysis [12] suggested that ADT without estrogen increases the risk of thromboembolic events. A database study also showed that ADT significantly increased the risk of thromboembolic events [13]. A Swedish database study [14] also showed that the risk of thromboembolic events was increased in patients with prostate cancer. These studies focused on cardiovascular events. Teoh et al. [15] reported on 452 Chinese men with prostate cancer treated primarily with radical prostatectomy or radiotherapy and compared the risk of acute myocardial infarction (AMI) in patients who were given ADT with those who were not given any. The ADT group was associated with an increased risk of AMI $(P=0.004)$ when compared to the non-ADT group. The use of ADT (HR: 6.78, 95\% confidence interval (CI): $1.31-35.05, P=0.022$ ) was a significant risk factor for AMI after multivariate Cox regression analysis. A retrospective study was then conducted by Teoh et al. [16] on 452 Chinese patients with prostate cancer. There were no statistically significant associations in the patients between administration of ADT and the development of ischemic stroke upon Kaplan-Meier analysis. However, upon multivariate Cox regression analysis, the use of $\mathrm{ADT}$ was a risk factor for ischemic stroke (HR: 3.32, 95\% CI: $1.14-9.67, P=0.028$ ).

There are limited data about ischemic stroke and ADT in prostate cancer patients. The aim of our study was to investigate the safety of ADT as reflected by outcomes of ischemic stroke and mortality in a nationwide cohort study involving patients with prostate cancer using the Taiwan National Health Insurance Research Databases (NHIRD).

\section{Methods \\ Database}

A medical claims database, the NHIRD, was created for research and includes outpatient and inpatient claims data. The database includes information regarding each patient's age, gender, diseases, prescription drugs, and medical expenditures. This study was a population-based retrospective cohort study. Our data were retrieved from the Longitudinal Health Insurance Database 2005, which includes data from 1,000,000 beneficiaries collected from 1997 to 2013. In this observational and analytical study, the database was derived from the NHIRD, and the data were deidentified secondary data that were released for research purposes. The study was reviewed and approved by the Institutional Review Board (IRB) of Kaohsiung Medical University Hospital. According to both the NHIRD and hospital regulations, informed consent from the patients was waived for this retrospective cohort study because the information was deidentified secondary data. All procedures were in accordance with the principles of the Declaration of Helsinki.

\section{Study population}

We identified individuals older than 20 years of age who were newly diagnosed with prostate cancer (International Classification of Diseases, ninth revision, Clinical Modification [ICD-9-CM]: 185) between January 1, 2005, and December 31, 2012. Patients without certification for catastrophic illness were excluded. The index date was assigned as the date of diagnosis of prostate cancer.

We excluded patients who were diagnosed with other cancers before the index date and those who took any second-line ADT drugs (high dose bicalutamide, diethylstilbestrol, and medroxyprogesterone) within 180 days after the index date. Furthermore, patients who experienced an ischemic stroke or a transient ischemic stroke before the index date were excluded from our observational populations. We further excluded patients who had other cancer diagnoses, those who had received chemotherapy before the index date, those with a history of ischemic stroke or ADT exposure before the index date, and patients who had received second-line ADT.

\section{Drug use}

Patients with prostate cancer who received at least one prescription for ADT within 6 months after the index date were defined as the ADT user group. Patients who did not receive at least one prescription for ADT within 6 months after the index date were defined as the ADT nonuser group. Each patient was followed until the first occurrence of one of the primary outcome measures (ischemic stroke or death), until they started receiving ADT if they were in the ADT user group, or until December 31, 2013.

\section{Comorbidities and medications}

Patient comorbidities were identified according to diagnostic codes for each inpatient or outpatient diagnosis 1 year before the index date. Comorbidities included diabetes mellitus, hypertension, dyslipidemia, ischemic heart disease, congestive heart failure, chronic obstructive pulmonary disease (COPD), atrial fibrillation, thyroid 
disease, chronic kidney disease, and liver diseases. The demographic characteristics of the patients were also assessed at baseline.

The use of concomitant drugs was identified according to claimed prescriptions for one year before the index date. These drugs included angiotensin-converting enzyme inhibitors (ACEIs), angiotensin II receptor blockers (ARBs), diuretics, beta blockers, alpha blockers, calcium channel blockers (CCBs), 3-hydroxy-3-methylglutaryl-coenzyme A (HMG-CoA) reductase inhibitors (statins), antiplatelet drugs, nonsteroidal anti-inflammatory drugs (NSAIDs), vitamin $\mathrm{K}$ antagonists, and diabetes medications.

\section{Study endpoints}

The composite primary endpoint was a combination of all-cause mortality or ischemic stroke. We also analyzed all-cause mortality and ischemic stroke as individual endpoints. For patients who visited the emergency department due to ischemic stroke, hospitalization due to ischemic stroke with a primary or secondary diagnosis code or hospitalization for carotid artery stent placement was defined as an ischemic stroke event.

\section{Statistical analysis}

We aimed to describe the demographics including the ages, comorbidities, and concomitant medication use of prostate cancer patients with and without ADT exposure. For the baseline characteristics, continuous variables are presented as the means and standard deviations and were compared by Student's t-tests. Discrete variables are presented as counts and percentages, and among patients with and without ADT exposure, these variables were compared by chi-square tests. Values of $P<0.05$ were considered statistically significant in all statistical analyses.

We performed 1:1 propensity score (PS) matching on each sample. The variables including age groups and comorbidities were used to calculate the predicted probability from a logistic regression model. A Kaplan-Meier curve was used to estimate the differences between patients who did and did not use ADT after PS matching and was assessed by the log-rank test. A Cox proportional hazards model was used to assess the association of outcomes between patients with and without ADT exposure during the follow-up period. Models were adjusted for age group, comorbidities, and concomitant medication use. To assess the robustness of the outcomes, sensitivity analysis for ischemic stroke was performed in a competing risk model, and subgroup analysis was performed for ADT use (over one year and less than one year). All data management procedures and statistical analyses were performed using SAS software, version 9.4 (SAS Institute, Cary, NC, USA). The research was approved by the IRB of Kaohsiung Medical University Hospital (KMUHIRB-EXEMPT-20170011).

\section{Results}

We enrolled 1588 patients older than 20 years with prostate cancer. A total of 1292 patients met the inclusion and exclusion criteria. We performed 1:1 PS matching of these patients, and 272 patients were included for further analysis. Figure 1 shows a diagram of patient enrollment.

Table 1 shows the patient characteristics before and after PS matching. The mean patient age was approximately 70 years, and more than one-tenth of the patients were older than 80 years. The five most common comorbidities in order were hypertension, dyslipidemia, COPD, ischemic heart disease and diabetes. The three most common medications used were NSAIDs, alpha blockers and CCBs.

Table 2 shows the outcomes of ADT users and nonusers with prostate cancer after PS matching. The primary composite outcome was the time to any cause of death or to ischemic stroke. There was no significant difference in primary composite outcome among ADT users and nonusers with prostate cancer. In terms of any cause of mortality, prostate cancer patients who received ADT had a higher mortality rate than those who did not receive ADT, and the adjusted hazard ratio was 1.907 (95\% CI: $1.278-2.844 ; P=0.0016$ ) after adjusting for age, diabetes mellitus, ischemic heart disease, congestive heart failure, COPD, hypertension, dyslipidemia, thyroid disease, atrial fibrillation, liver disease, renal disease and comedication, as shown in Table 1 . The results showed that patients treated with ADT during follow-up did not have a significantly higher risk of ischemic stroke than those not treated with ADT (crude HR: 1.147, $P=$ 0.3451; adjusted HR: 0.812, $P=0.3291$ ).

In subgroup analysis, the patients who used ADT over one year did not have a significantly increased risk of ischemic stroke than the ADT nonuser patients (adjusted HR: $1.132, P=0.5745)$; there was also no significant difference in ischemic stroke risk between patients who used ADT for less than a year and those who did not use ADT (adjusted HR: $1.217(p=0.2311))$. Furthermore, we also repeated the analysis for ischemic stroke in a competing risk model (adjusted HR: 0.759, $P=0.2050$ ), which also showed that ADT use did not significantly increase the risk of ischemic stroke.

Figure 2 shows the probability of freedom from ischemic stroke after propensity score matching stratified by ADT users and nonusers. There was no significant difference in ischemic stroke between ADT users and nonusers. $(P=0.3805)$.

\section{Discussion}

In prostate cancer patients treated with ADT, the severity conferred by endocrine treatment was greater than 
Adults $\geq 20 \mathrm{y} / 0$ with prostate cancer in 2005

to $2012, n=1,588$

1. Exclude other cancer diagnosis or receive chemotherapy before index date in study. $\mathrm{n}=95$

2. Exclude patients with a stroke diagnosis before the index date. $\mathrm{n}=175$

3. Exclude patients with ADT exposure before the index date. $\mathrm{n}=6$

4. Exclude patients treated with second-line ADT (high dose bicalutamide, diethylstilbestrol, medroxyprogesterone. $\mathrm{N}=20$

Adults $\geq 20 \mathrm{y} / 0$ with prostate cancer in 2005 to $2012, \mathrm{n}=1,292$

Patients treated with ADT within 180 after index date. $n=1,019$

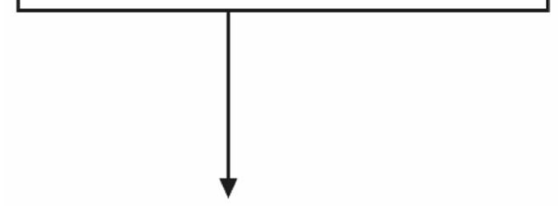

PS -matching

$1: 1$
Patients treated with ADT within

180 after index date. $n=272$
Patients not treated with ADT

within 180 after index date. $n=272$

Fig. 1 Study flowchart 
Table 1 Baseline characteristics of the prostate cancer patients after matching

\begin{tabular}{|c|c|c|c|c|c|c|c|c|c|c|}
\hline \multirow[b]{3}{*}{ Characteristic } & \multirow{2}{*}{\multicolumn{2}{|c|}{$\begin{array}{l}\text { ADT } \\
\text { Nonusers } \\
(n=273)\end{array}$}} & \multirow{2}{*}{\multicolumn{2}{|c|}{$\begin{array}{l}\text { ADT users } \\
(n=1019)\end{array}$}} & \multirow[b]{3}{*}{$p$-value } & \multicolumn{4}{|l|}{ PS Matching } & \multirow[b]{3}{*}{$p$-value } \\
\hline & & & & & & \multirow{2}{*}{$\begin{array}{l}\text { ADT nonusers }(n=272) \\
n\end{array}$} & \multicolumn{3}{|c|}{ ADT users $(n=272)$} & \\
\hline & $n$ & $\%$ & $n$ & $\%$ & & & $\%$ & $n$ & $\%$ & \\
\hline$\overline{\text { Age, mean SD }}{ }^{\mathrm{a}}$ & 69.8 & 8.59 & 73.7 & 7.86 & $<0.001$ & 69.8 & 8.59 & 70.3 & 8.71 & 0.488 \\
\hline Age stratification & & & & & $<0.001$ & & & & & 0.978 \\
\hline$\leq 60$ & 39 & 14.3 & 73 & 7.2 & & 38 & 14.0 & 39 & 14.3 & \\
\hline $61-70$ & 98 & 35.9 & 247 & 24.2 & & 98 & 36.0 & 96 & 35.3 & \\
\hline $71-80$ & 107 & 39.2 & 481 & 47.2 & & 107 & 39.3 & 105 & 38.6 & \\
\hline$>80$ & 29 & 10.6 & 218 & 21.4 & & 29 & 10.7 & 32 & 11.8 & \\
\hline \multicolumn{11}{|l|}{ Comorbidity } \\
\hline Diabetes mellitus & 50 & 18.3 & 221 & 21.7 & 0.224 & 50 & 18.4 & 47 & 17.3 & 0.737 \\
\hline Ischemic heart disease & 52 & 19.1 & 205 & 20.1 & 0.694 & 52 & 19.1 & 53 & 19.5 & 0.914 \\
\hline Congestive heart failure & 16 & 5.9 & 76 & 7.5 & 0.362 & 16 & 5.9 & 16 & 5.9 & 1.000 \\
\hline COPD & 59 & 21.6 & 249 & 24.4 & 0.331 & 59 & 21.7 & 53 & 19.5 & 0.525 \\
\hline Hypertension & 139 & 50.9 & 565 & 55.5 & 0.182 & 138 & 50.74 & 131 & 48.2 & 0.548 \\
\hline Dyslipidemia & 75 & 27.5 & 216 & 21.2 & 0.028 & 74 & 27.2 & 74 & 27.2 & 1.000 \\
\hline Thyroid disease & 6 & 2.2 & 17 & 1.7 & 0.557 & 6 & 2.2 & 5 & 1.8 & 0.761 \\
\hline Atrial fibrillation & 4 & 1.5 & 19 & 1.9 & 0.658 & 4 & 1.5 & 2 & 0.7 & 0.412 \\
\hline Liver disease & 32 & 11.7 & 117 & 11.5 & 0.912 & 32 & 11.7 & 32 & 11.7 & 1.000 \\
\hline Renal disease & 14 & 5.1 & 72 & 7.1 & 0.254 & 14 & 5.2 & 9 & 3.3 & 0.287 \\
\hline \multicolumn{11}{|l|}{ Comedications } \\
\hline ACEIS & 34 & 12.5 & 158 & 15.5 & 0.281 & 34 & 12.5 & 38 & 14.0 & 0.613 \\
\hline ARBs & 34 & 12.5 & 163 & 16.0 & 0.148 & 33 & 12.1 & 43 & 15.8 & 0.216 \\
\hline Beta blockers & 76 & 27.8 & 261 & 25.6 & 0.457 & 75 & 27.6 & 70 & 25.7 & 0.628 \\
\hline CCBs & 108 & 39.6 & 419 & 41.1 & 0.642 & 107 & 39.3 & 100 & 36.8 & 0.537 \\
\hline Alpha blockers & 80 & 29.3 & 381 & 37.4 & 0.013 & 80 & 29.4 & 102 & 37.5 & 0.046 \\
\hline NSAIDs & 231 & 84.6 & 837 & 82.1 & 0.337 & 230 & 84.6 & 227 & 83.5 & 0.726 \\
\hline Statins & 46 & 16.9 & 122 & 12.0 & 0.033 & 45 & 16.5 & 37 & 13.6 & 0.338 \\
\hline Antiplatelet drugs & 52 & 19.1 & 207 & 20.3 & 0.643 & 52 & 19.1 & 49 & 18.0 & 0.741 \\
\hline Anticoagulants & 2 & 0.7 & 12 & 1.2 & 0.528 & 2 & 0.7 & 5 & 1.8 & 0.254 \\
\hline Antidiabetic drugs & 38 & 13.9 & 183 & 18.0 & 0.116 & 38 & 14.0 & 44 & 16.2 & 0.472 \\
\hline
\end{tabular}

that of the other treatments. These prostate cancer patients with more advanced disease may experience a poor prognosis and high mortality.

Albertsen et al. [17] performed six phase 3 prospective randomized trials to show cardiovascular morbidity following initiation of gonadotropin-releasing hormone agonists compared with an antagonist. The risk of cardiac events within 1 year of initiating therapy was lower in patients received gonadotropin-releasing hormone antagonists compared with gonadotropinreleasing hormone agonists (HR: 0.44, 95\% CI: $0.26-$ $0.74, P=0.002)$.
Teoh et al. [18] found that surgical castration was associated with an increased risk of cardiovascular thrombotic events when compared to gonadotropin-releasing hormone agonists. After multivariate Cox regression analysis, age, hyperlipidemia, and surgical castration were significant risk factors of cardiovascular thrombotic events. Chung et al. [19] also used the NHIRD and found that there was no significant difference in the risk of stroke in patients with prostate cancer who did and did not receive ADT in Taiwan, after adjusting for potential confounders. Some physiological and genetic differences have been observed in different ethnicities. The 
Table 2 Cox proportional hazard models comparing ADT users and nonusers with prostate cancer after matching

\begin{tabular}{|c|c|c|c|c|c|c|c|c|c|c|}
\hline \multirow[t]{3}{*}{ Outcomes } & \multicolumn{5}{|c|}{ Before Matching } & \multicolumn{5}{|c|}{ Propensity Score Matching } \\
\hline & \multirow{2}{*}{$\begin{array}{l}\text { ADT Nonuser } \\
\text { Event }\end{array}$} & \multicolumn{4}{|l|}{ ADT user } & \multirow{2}{*}{$\begin{array}{l}\text { ADT Nonuser } \\
\text { Event }\end{array}$} & \multirow{2}{*}{$\begin{array}{l}\text { ADT user } \\
\text { Event }\end{array}$} & \multirow[t]{2}{*}{ Adjusted $\mathrm{HR}^{a}$} & \multirow[t]{2}{*}{$95 \% \mathrm{Cl}$} & \multirow[t]{2}{*}{$p$-value } \\
\hline & & Event & Adjusted $H R^{a}$ & $95 \% \mathrm{Cl}$ & $p$-value & & & & & \\
\hline Primary outcome & $82(30.0)$ & $499(49.87)$ & 1.332 & $1.051-1.687$ & $0.0177^{*}$ & $82(30.2)$ & $114(41.9)$ & 1.192 & $0.885-1.605$ & 0.2471 \\
\hline $\begin{array}{l}\text { Any cause of } \\
\text { death }\end{array}$ & $37(13.6)$ & $375(36.8)$ & 2.107 & $1.499-2.900$ & $<0.0001^{*}$ & 37 (13.6) & $85(31.3)$ & 1.907 & $1.278-2.844$ & $0.0016^{*}$ \\
\hline Ischemic stroke & $49(18.0)$ & $203(19.9)$ & 0.973 & $0.709-1.334$ & 0.8640 & $49(18.0)$ & $45(16.5)$ & 0.812 & $0.534-1.234$ & 0.3291 \\
\hline
\end{tabular}

$H R$ hazard ratio, $\mathrm{Cl}$ confidence interval

* $p$-value $<0.05$

a adjusted variables included age group, comorbidities and comedications

UGT2B gene and androgen receptor genetic polymorphisms, namely, the CAG repeat length polymorphism, may possibly lead to differences in the cardiovascular risk between Asians and Caucasians. Asians have longer CAG repeat lengths than Caucasians, and testosterone suppression with ADT may lead to higher cardiovascular risk in Asians than in Caucasians, although we need more evidence to support this hypothesis [20].

Prostate cancer patients who received ADT seemed to be at an increased risk of having thromboembolic events, including arterial embolism, pulmonary embolism or deep venous thrombosis. A recent meta-analysis including 10 studies found that ADT increased the risk of thromboembolic events with a risk ratio of 1.43 (95\% CI: 1.15-1.77, $P=0.001$ ) [12]. Another database study analyzed 58,466 patients treated with ADT [13] and found that ADT was associated with an increased risk of thromboembolic events (adjusted hazard ratio $=1.56$; 95\% CI: $1.50-1.61 ; P<0.0001)$.

A Swedish database study analyzed 30,642 men with prostate cancer on endocrine therapy and found increased risks of deep vein thrombosis (standardized incidence ratio $(\mathrm{SIR})=2.48,95 \% \mathrm{CI}=2.25-2.73)$ and pulmonary embolism $(\mathrm{SIR}=1.95,95 \% \mathrm{CI}=1.81-2.15)$ but did not increase in arterial embolism $(\mathrm{SIR}=1.00$, 95\% CI $=0.82-1.20)$ [14].

Malignant disease is associated with the risk of thromboembolic disease and has long been recognized in clinical practice. Previous studies have reported this risk of thromboembolism in patients with prostate cancer, but these studies did not focus on ischemic stroke [21-23]. Other malignant diseases, such as breast cancer,

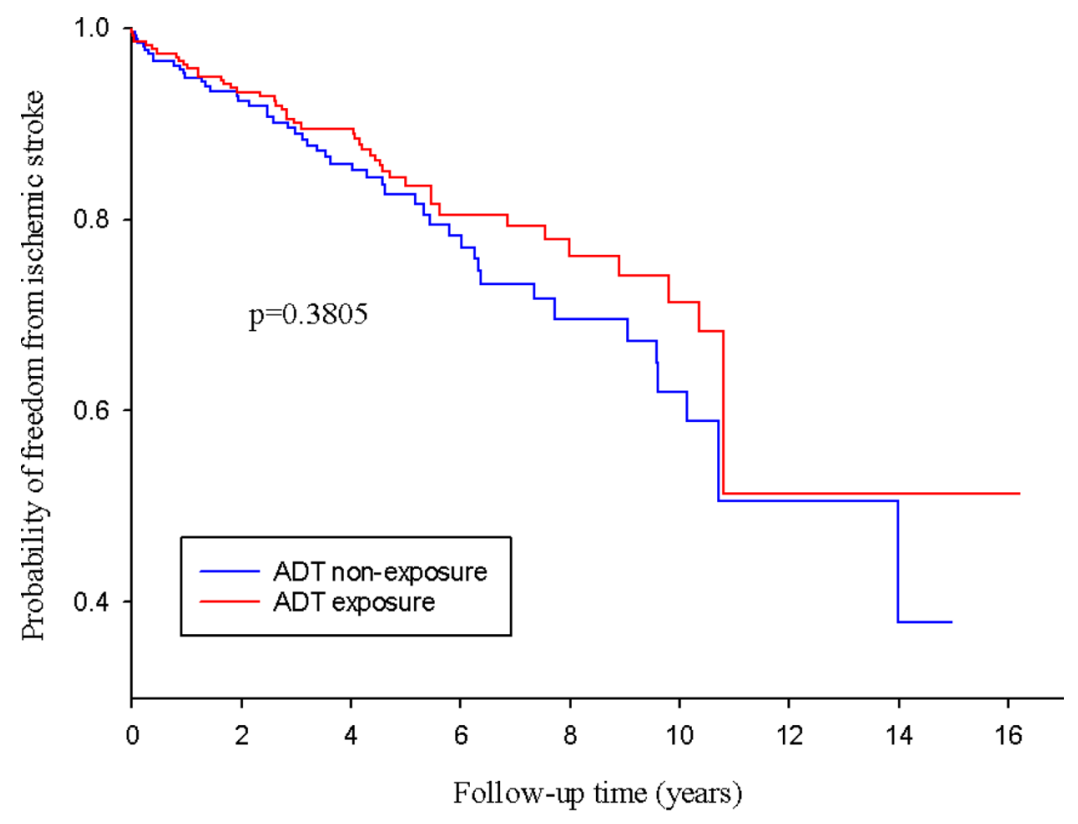

$\begin{array}{llllllllll}\text { ADT exposure } & 272 & 217 & 166 & 80 & 57 & 33 & 12 & 4 & 1 \\ \text { ADT non-exposure } & 272 & 185 & 132 & 71 & 45 & 24 & 14 & 6 & 0\end{array}$

Fig. 2 Probability of freedom from ischemic stroke following propensity score matching stratified by ADT exposure and non-exposure 
malignant gliomas and ovarian cancer, are also associated with a high risk of thromboembolic events affecting 1 to $28 \%$ of patients [22].

Graus et al. [24] conducted an autopsy study to assess the prevalence of pathological findings of cerebrovascular disease in cancer patients and found that $14.6 \%$ of patients had pathologic evidence of cerebrovascular disease, and $7.4 \%$ had clinical symptoms of cerebrovascular disease. The risk factors for cerebrovascular disease were obscured by pathophysiologic factors associated with malignancy, including direct effects of the malignancy, hypercoagulable state, susceptibility to infections, treatment medication and the process of treatment or diagnosis. Cestari et al. [25] used a registry database to evaluate the incidence and type of strokes in cancer patients and showed that the three most common primary cancers were lung cancer (30\%), brain cancer (9\%) and prostate cancer (9\%). The most common cause of stroke was embolic stroke, occurring in $52(54 \%)$ patients. Forty-four (46\%) patients had nonembolic stroke. The mechanism of stroke was partially due to hypercoagulability, and atherosclerosis only explained 22\% of strokes in cancer patients. Outcome s were primarily decided by the underlying cancer stage.

Bosco et al. [26] included 8 studies in a meta-analysis and found a positive association between ADT and the risk of cardiovascular disease. In their meta-analysis, most studies focused on myocardial infarction, and one study focused on ADT and ischemic stroke. Azoulay et al. [27] used the General Practice Research Database, a primary care database from the United Kingdom, to conduct a nested casecontrol analysis and found that ADT increased the risk of stroke or transient ischemic attacks in patients with prostate cancer. In contrast to their study, our study found that ADT was not associated with the risk of ischemic stroke in prostate cancer. There are some differences between their study and our study. First, although both retrospective studies used a database, our study design was a cohort study, while Azoulay et al. used nested case-control analysis. In the presence of competing risks, the use of a nested case-control design results in greater bias compared to the use of a cohort design whether treatment occurs at baseline or varies over time and there is a single outcome. The cohort design has greater precision and a lower mean squared error in all scenarios [28]. Second, we used PS matching and adjusted for more cardiovascular medications. Third, their definition of stroke included hemorrhagic stroke because the stroke subtype (ischemic vs hemorrhagic) cannot be differentiated from the General Practice Research Database in the United Kingdom. Fourth, they excluded patients with evidence of metastases at diagnosis. We enrolled patients with all stages of prostate cancer.

Our population-based cohort study has some advantages. First, this was a large population-based cohort of patients with prostate cancer who were followed for up to 9 years. Second, the National Health Insurance program in Taiwan is compulsory for all citizens and covers almost all services that can be provided by a healthcare system. Third, we enrolled all patients with prostate cancer who were treated with ADT. Fourth, in Taiwan, patients with malignant diseases have catastrophic illness certification and do not need to pay a copayment for outpatient or inpatient care. Their applications are formally reviewed by more than two physicians and have a low probability of misdiagnosis. The prescriptions in Taiwan are also written by specialists (urologists or oncologists); thus, patients have a low probability of being misclassified as ADT users or ADT nonusers.

There are some limitations to our study. The NHIRD lacks some confounders, such as body mass index, cancer stage and smoking status. Therefore, we were unable to adjust for these confounders. This study evaluated the association of ADT and ischemic stroke in all prostate cancer patients regardless of the stage of disease. Second, there may be some asymptomatic ischemic stroke patients who were not diagnosed. These patients did not receive comprehensive routine brain magnetic resonance imaging examinations; thus, small stroke lesions may have been omitted. It is also possible that a rapidly massive fatal ischemic stroke in a prostate cancer patient with advanced disease could have been interpreted as the fatal end-stage of prostate cancer and therefore was not classified as our interest outcome of ischemic stroke. However, we believe that only a small number of cases may have been misclassified in this condition. Babiker et al. [29] suggested that prostasomes, submicron secretory granules from the prostate gland, evoke blood coagulation in prostate cancer and result in thromboembolic disease. Other studies [30, 31] have also shown a link between testosterone and enhancement of fibrinolytic inhibition via increased synthesis of the plasminogen activator inhibitor plasminogen activator inhibitor-1, but our study found that the risk of ischemic stroke did not significantly increase in prostate cancer patients who received ADT. We may need a prospective study with a large sample size to assess the association between ischemic stroke and ADT, and whether there is a different thromboembolic mechanism between myocardial infarction and ischemic stroke in prostate cancer patients receiving ADT needs to be further investigated.

\section{Conclusions}

In this nationwide population-based study, we compared the risk of ischemic stroke between patients with prostate cancer who were and were not treated with ADT. Our study showed that patients with prostate cancer who received ADT did not have an increased risk of ischemic stroke, but they did have an increased risk of mortality, which may result from confounding by indication. 


\section{Abbreviations}

ACEls: Angiotensin-converting enzyme inhibitors; ADT: Androgen deprivation therapy; ARBs: Angiotensin II receptor blockers; CCBs: Calcium channel blockers; Cl: Confidence interval; COPD: Chronic obstructive pulmonary disease; HR: Hazard ratio; NHIRD: National Health Insurance Research Databases; NSAIDs: Nonsteroidal anti-inflammatory drugs; PS: Propensity score; SIR: Standardized incidence ratio

\section{Acknowledgements}

This study was based in part on data from the NHIRD provided by the Bureau of National Health Insurance (BNHI) of the Department of Health and managed by the National Health Research Institute. The conclusions presented in this study are those of the authors and do not necessarily reflect the views of the BNHI, the Department of Health, or the National Health Research Institute. This work was supported by the Kaohsiung Medical University Hospital (KMUH106-M616).

\section{Authors' contributions}

KML: wrote the manuscript; interpretation of findings and critical edit of manuscript; CCK: conceived and designed the study; collected and analyzed the data; contributed with critical reviews of the data analyses, CYC: conceived and designed the study; collected and analyzed the data; contributed with critical reviews of the data analyses and interpretation of the findings; reviewed the manuscript; supervised the study; YBH: contributed with critical reviews of the data analyses, interpretation of the findings and critical edits of the manuscript; reviewed the manuscript. All authors have read and approved the final manuscript.

\section{Funding}

The design of the study and collection, analysis, and interpretation of data and editorial writing support was funded by the Kaohsiung Medical University Hospital (KMUH106-M616)

\section{Availability of data and materials}

The data that support the findings of this study are available from the NHIRD, but restrictions apply to the availability of these data, which were used under license for the current study and so are not publicly available. Data are, however, available from the authors upon reasonable request and with permission from the NHIRD.

\section{Ethics approval and consent to participate}

The research was approved by an IRB from Kaohsiung Medical University Hospital (KMUHIRB-EXEMPT-20170011), which also permitted waving of the need for consent.

\section{Consent for publication}

Not applicable.

\section{Competing interests}

The authors declare that they have no competing interests.

\section{Author details \\ 'Department of Internal Medicine, Chi Mei Medical Center, Chiali, Taiwan. ${ }^{2}$ School of Pharmacy, Master Program in Clinical Pharmacy, Kaohsiung Medical University, No. 100, Shihcyuan 1st Rd., Sanmin District, Kaohsiung City 80708, Taiwan, Republic of China. ${ }^{3}$ Department of Pharmacy, Kaohsiung Medical University Hospital, Kaohsiung, Taiwan. ${ }^{4}$ Department of Medical Research, Kaohsiung Medical University Hospital, Kaohsiung, Taiwan.}

\section{Received: 18 April 2019 Accepted: 20 December 2019}

Published online: 30 December 2019

\section{References}

1. Heidenreich A, Bastian PJ, Bellmunt J, et al. EAU guidelines on prostate cancer. Part II: treatment of advanced, relapsing, and castration-resistant prostate cancer. Eur Urol. 2014;65:467-79.

2. Hussain $\mathrm{M}$, Tangen $\mathrm{CM}$, Berry $\mathrm{DL}$, et al. Intermittent versus continuous androgen deprivation in prostate cancer. N Engl J Med. 2013;368:1314-25.

3. Gay HA, Sanda MG, Liu J, et al. External beam radiation therapy or brachytherapy with or without short-course Neoadjuvant androgen deprivation therapy: results of a multicenter, prospective study of quality of life. Int J Radiat Oncol Biol Phys. 2017;98:304-17.

4. Smith MR, Lee WC, Brandman J, et al. Gonadotropin-releasing hormone agonists and fracture risk: a claims-based cohort study of men with nonmetastatic prostate cancer. J Clin Oncol. 2005;23:7897-903.

5. Irani J, Salomon L, Oba R, et al. Efficacy of venlafaxine, medroxyprogesterone acetate, and cyproterone acetate for the treatment of vasomotor hot flushes in men taking gonadotropin-releasing hormone analogues for prostate cancer: a double-blind, randomised trial. Lancet Oncol. 2010;11:147-54.

6. Smith MR, Lee H, Fallon MA, Nathan DM. Adipocytokines, obesity, and insulin resistance during combined androgen blockade for prostate cancer. Urology. 2008;71:318-22

7. Keating NL, O'Malley AJ, Freedland SJ, Smith MR. Diabetes and cardiovascular disease during androgen deprivation therapy: observational study of veterans with prostate cancer. J Natl Cancer Inst. 2010;102:39-46.

8. Saigal CS, Gore JL, Krupski TL, et al. Androgen deprivation therapy increases cardiovascular morbidity in men with prostate cancer. Cancer. 2007;110: 1493-500.

9. Tsai HK, D'Amico AV, Sadetsky N, et al. Androgen deprivation therapy for localized prostate cancer and the risk of cardiovascular mortality. J Natl Cancer Inst. 2007:99:1516-24

10. Nanda A, Chen MH, Braccioforte $\mathrm{MH}$, et al. Hormonal therapy use for prostate cancer and mortality in men with coronary artery disease-induced congestive heart failure or myocardial infarction. JAMA. 2009;302:866-73.

11. Nguyen $P L$, Je $Y$, Schutz FA, et al. Association of androgen deprivation therapy with cardiovascular death in patients with prostate cancer: a metaanalysis of randomized trials. JAMA. 2011;306:2359-66.

12. Nead KT, Boldbaatar N, Yang DD, et al. Association of Androgen Deprivation Therapy and Thromboembolic Events: a systematic review and metaanalysis. Urology. 2018;114:155-62.

13. Ehdaie B, Atoria CL, Gupta A, et al. Androgen deprivation and thromboembolic events in men with prostate cancer. Cancer. 2012;118:3397-406.

14. Van Hemelrijck M, Adolfsson J, Garmo H, et al. Risk of thromboembolic diseases in men with prostate cancer: results from the population-based PCBaSe Sweden. Lancet Oncol. 2010;11:450-8.

15. Teoh JY, Chan SY, Chiu PK, Poon DM, Cheung HY, Hou SS, et al. Risk of acute myocardial infarction after androgendeprivation therapy for prostate cancer in a Chinese population. BJU Int. 2015;116:382-7.

16. Teoh JY, Chiu PK, Chan SY, Poon DM, Cheung HY, Hou SS, et al. Risk of ischemic stroke after androgen deprivation therapy for prostate cancer in the Chinese population living in Hong Kong. Jpn J Clin Oncol. 2015;45: 483-7.

17. Albertsen PC, Klotz L, Tombal B, Grady J, Olesen TK, Nilsson J. Cardiovascular morbidity associated with gonadotropin releasing hormone agonists and an antagonist. Eur Urol. 2014;65:565-73

18. Teoh JY, Chan SY, Chiu PK, Poon DM, Cheung HY, Hou SS, Ng CF. Risk of cardiovascular thrombotic events after surgical castration versus gonadotropin-releasing hormone agonists in Chinese men with prostate cancer. Asian J Androl. 2015;17:493-6.

19. Chung SD, Chen YK, Wu FJ, Lin HC. Hormone therapy for prostate cancer and the risk of stroke: a 5-year follow-up study. BJU Int. 2012;109:1001-5.

20. Teoh JY, Ng CF. Cardiovascular risk after androgen deprivation therapy for prostate cancer: an Asian perspective. Int Urol Nephrol. 2016;48:1429-35.

21. Seaman HE, Langley SE, Farmer RD, de Vries CS. Venous thromboembolism and cyproterone acetate in men with prostate cancer: a study using the general practice research database. BJU Int. 2007;99:1398-403.

22. Secin FP, Jiborn T, Bjartell AS. Multi-institutional study of symptomatic deep venous thrombosis and pulmonary embolism in prostate cancer patients undergoing laparoscopic or robot-assisted laparoscopic radical prostatectomy. Eur Urol. 2008;53:134-45.

23. Lee AY, Levine MN. Venous thromboembolism and cancer: risks and outcomes. Circulation. 2003;107:117-21.

24. Graus F, Rogers LR, Posner JB. Cerebrovascular complications in patients with cancer. Medicine (Baltimore). 1985;64:16-35.

25. Cestari DM, Weine DM, Panageas KS, Segal AZ, DeAngelis LM. Stroke in patients with cancer: incidence and etiology. Neurology. 2004;62:2025-30.

26. Bosco C, Bosnyak Z, Malmberg A, Adolfsson J, Keating NL, Van Hemelrijck M. Quantifying observational evidence for risk of fatal and nonfatal cardiovascular disease following androgen deprivation therapy for prostate cancer: a meta-analysis. Eur Urol. 2015;68:386-96. 
27. Azoulay L, Yin H, Benayoun S, Renoux C, Boivin J-F, Suissa S. Androgendeprivation therapy and the risk of stroke in patients with prostate cancer Eur Urol. 2011;60:1244-50.

28. Austin PC, Anderson GM, Cigsar C, Gruneir A. Comparing the cohort design and the nested case-control design in the presence of both time-invariant and time-dependent treatment and competing risks: bias and precision. Pharmacoepidemiol Drug Saf. 2012;21:714-24.

29. Babiker AA, Ekdahl KN, Nilsson B, Ronquist G. Prothrombotic effects of prostasomes isolated from prostatic cancer cell lines and seminal plasma. Semin Thromb Hemost. 2007;33:80-6.

30. Toorians AW, Thomassen MC, Zweegman S. Venous thrombosis and changes of hemostatic variables during cross-sex hormone treatment in transsexual people. J Clin Endocrinol Metab. 2003;88:5723-9.

31. Winkler UH. Effects of androgens on haemostasis. Maturitas. 1996;24:147-55.

\section{Publisher's Note}

Springer Nature remains neutral with regard to jurisdictional claims in published maps and institutional affiliations.

Ready to submit your research? Choose BMC and benefit from:

- fast, convenient online submission

- thorough peer review by experienced researchers in your field

- rapid publication on acceptance

- support for research data, including large and complex data types

- gold Open Access which fosters wider collaboration and increased citations

- maximum visibility for your research: over $100 \mathrm{M}$ website views per year

At BMC, research is always in progress.

Learn more biomedcentral.com/submissions 